\title{
Cardiac Arrhythmias Classification Method Based on MUSIC, Morphological Descriptors, and Neural Network
}

\author{
Ahmad R. Naghsh-Nilchi and A. Rahim Kadkhodamohammadi \\ Department of Computer Engineering, University of Isfahan, Isfahan 81746-73441, Iran \\ Correspondence should be addressed to Ahmad R. Naghsh-Nilchi, nilchi@yahoo.com
}

Received 6 June 2008; Revised 27 September 2008; Accepted 12 December 2008

Recommended by Tan Lee

An electrocardiogram (ECG) beat classification scheme based on multiple signal classification (MUSIC) algorithm, morphological descriptors, and neural networks is proposed for discriminating nine ECG beat types. These are normal, fusion of ventricular and normal, fusion of paced and normal, left bundle branch block, right bundle branch block, premature ventricular concentration, atrial premature contraction, paced beat, and ventricular flutter. ECG signal samples from MIT-BIH arrhythmia database are used to evaluate the scheme. MUSIC algorithm is used to calculate pseudospectrum of ECG signals. The low-frequency samples are picked to have the most valuable heartbeat information. These samples along with two morphological descriptors, which deliver the characteristics and features of all parts of the heart, form an input feature vector. This vector is used for the initial training of a classifier neural network. The neural network is designed to have nine sample outputs which constitute the nine beat types. Two neural network schemes, namely multilayered perceptron (MLP) neural network and a probabilistic neural network (PNN), are employed. The experimental results achieved a promising accuracy of $99.03 \%$ for classifying the beat types using MLP neural network. In addition, our scheme recognizes NORMAL class with 100\% accuracy and never misclassifies any other classes as NORMAL.

Copyright ( 2008 A. R. Naghsh-Nilchi and A. R. Kadkhodamohammadi. This is an open access article distributed under the Creative Commons Attribution License, which permits unrestricted use, distribution, and reproduction in any medium, provided the original work is properly cited.

\section{INTRODUCTION}

Most physiological activities consist of certain signals that reflect the activities' nature and functions. These signals are of different types, such as biochemical signals in the form of neuron transition and hormone, physical signals in the form of pressure and temperature, and electrical signals in the form of voltage and current. Disease or biological system defects cause disorders in the function of physiological procedures as well as their corresponding signals. One could study the signal behaviors to identify the nature and type of disorders or diseases.

Heart is one of the most important organs of body and disorders in its function can cause serious problems for the patient. Arrhythmias are abnormal heartbeats. In fact, arrhythmias are heart diseases, caused by heart electricalconductive system disorders and heart diseases such as very slow (bradycardia) or very fast (tachycardia) heart functions and result in an inefficient pumping.
The heart state is generally reflected in the shape of ECG waveform and heart rate. They may contain important pointers to the nature of diseases afflicting the heart. However, because the signals are nonstationary in nature, the reflection may occur at random moments in the timescale (i.e., the disease symptoms are not present all the time, but could manifest at certain irregular intervals during the day). Therefore, for effective diagnostics, ECG pattern and heart rate variability have to be observed over several hours, which results in an enormous data volume. The study of such volume of data is, of course, tedious and time consuming. In addition, the possibility of the professional observer errors (or misreading) vital information is also high. Thus, computer-based analysis and classification of diseases could be proved to be very helpful in heart diagnostics [1].

Various computer-based methodologies for automatic diagnosis have been proposed by researchers; however, the entire process can generally be subdivided into a number of 
separate processing modules such as preprocessing, feature extraction/selection, and classification [2].

Signal acquisition, artifact removing, averaging, thresholding, and signal enhancement are the main operations in the course of preprocessing. Conventionally, ECG signal is measured on static condition since various types of noise including muscle artifact and electrode moving artifact are coupled in dynamic environment. To solve this problem, various noised signals are grouped into six categories by context estimation. Then neural network and genetic algorithm are used to effectively reconfigure noise reduction filter [3]. Digital filters are proposed to remove high- and low-frequency noises [4].

Another processing module is feature extraction. It is the determination of a feature or a feature vector from a pattern vector. The feature vector, which is comprised of the set of all features used to describe a pattern, is a reduced-dimensional representation of that pattern. The module of feature selection is an optional stage, whereby the feature vector is reduced in size including only what may be considered as the most relevant features required for discrimination, from the classification viewpoint. In the feature extraction stage, numerous different methods can be used so that several diverse features can be extracted from the same raw data. To a large extent, each feature can independently represent the original data, but none of them is totally perfect for practical applications. Moreover, there seems to be no simple way to measure relevance of the features for a pattern classification task. ECG features can be extracted in time-domain, in frequency-domain, or may be represented as statistical measures [5]. They can be based on ECG morphology and RR-intervals [6].

Jekova et al. [7] used 26 morphological descriptors representing information of the amplitude, area, specific interval durations, and measurements of the QRS vector in the vectorcardiographic (VCG) plane. Ceylan and Ozbay [8] applied principal component analysis and wavelet transform for feature extraction. Fourier and wavelet analyses are investigated for feature extraction [9]. Chazal and Reilly [10] Considered feature sets that include wavelet-based features, standard cardiology features, and features taken directly from time-domain samples of ECG beat. Yu and Chou [11] used independent component analysis to decompose ECG signals into weighted sum of basic components that are statistically mutual independent, and then the projections on these components, together with the RR interval constitute a feature vector.

The classification module is the final stage in automated diagnosis. It examines the input feature vector and based on its algorithmic nature produces a suggestive hypothesis. Fuzzy c-means clustering and multilayered perceptron are applied in [8]. Tadejko and Rakowski [6] developed a classifier with SOM and learning vector quantization algorithms. Yu and Chou [11] employed probabilistic neural network (PNN) and a back-propagation neural network, as classifiers. Jekova et al. [7] present a comparative study of the learning capacity and the classification abilities of four classification methods $-k$ th nearest neighbor rule, neural networks, discriminant analysis, and fuzzy logic. Chazal and
Reilly [10] compare the functions of linear, quadratic, and logistic discriminants as ECG classifiers.

In this paper, we evaluate the ability of multiple signal classification (MUSIC), morphological descriptor, and neural networks classifier to discriminate nine types of electrocardiogram beats. The MUSIC method enables us to estimate the spectrum of ECG signals with very high resolution under a low-signal-to-noise ratio (SNR) situation even if signal has a small data points [12-16].

\section{PROPOSED METHOD}

First, the signals are preprocessed by filtering and scaling to remove high frequency noise, enhance signal quality, and omit equipment and environment effects. Next, MUSIC algorithm is applied to calculate pseudospectrum of ECG signals, where the first 28 frequency samples of the pseudospectrum together with the variances of peak times and values are used as feature set. Finally, two different neural networks, including a probabilistic neural network and a multilayered perceptron neural network, are employed in this study. The experimental results demonstrate the effectiveness and efficiency of the proposed feature set and MLP neural network for ECG beat recognition.

\subsection{Preprocessing}

The objectives of preprocessing stage are the omission of high-frequency noise and the enhancement of signal quality to obtain appropriate features. Furthermore, we should remove equipment and environment influences on recorded signals. In this stage, patterns are filtered and then scaled.

\subsubsection{Filtering}

In the MIT/BIH arrhythmia database, the analog outputs of the playback unit are filtered to limit analog-to-digital converter saturation and for antialiasing, using a bandpass filter with a passband from 0.1 to $100 \mathrm{~Hz}$ relative to real time [17]. In this study, because of its simplicity and fidelity, an integer coefficient digital lowpass filter, proposed by Lo and Tang [4], was used to remove noise caused by power line interference, muscle tremors, and spikes:

$$
\mathrm{L}(\mathrm{z})=\frac{1-2 Z^{-6}+Z^{-12}}{1-2 Z^{-1}+Z^{-2}}
$$

The $3 \mathrm{~dB}$ point is at $20 \mathrm{~Hz}$, and the first side-lobe zero amplitude is at $60 \mathrm{~Hz}$. Therefore, power line interference at $60 \mathrm{~Hz}$ is completely eliminated, and high-frequency muscle tremor noise is minimized, which is predominately a result of the bandlimited (antialiased filtered) data in the MIT/BIH arrhythmia database [18].

The magnitude and phase characteristics are shown in Figure 1. One of the advantages of this filter structure is its linear phase. In Figure 2, the phase plot is shown in its wrapped form, where the phase is bound between $\pm \pi$. In its unwrapped form, the linear nature of the phase is evident. 


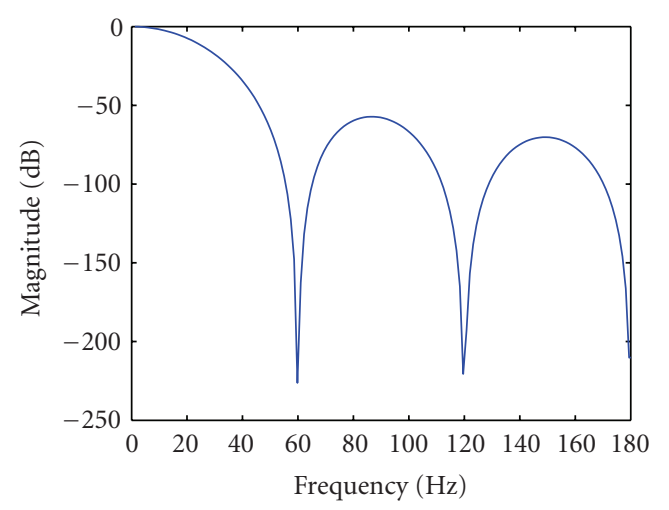

(a)

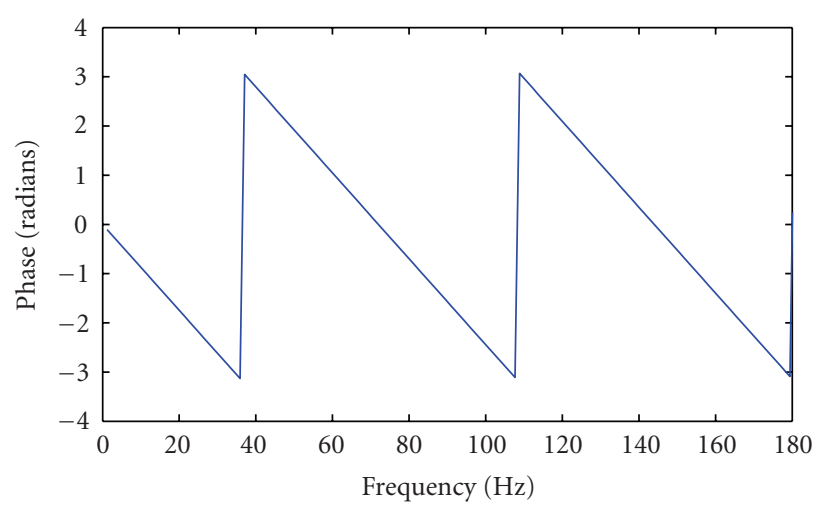

(b)

FIGURE 1: Magnitude and phase of lowpass filter.

\subsubsection{Scaling}

In order to reduce amplitude variations, each QRS segment is scaled to lie between 0 and 1 . Figure 2 shows a normal pattern and its processed form.

\subsection{Feature extraction}

\subsubsection{MUSIC algorithm}

Regarding several of the frequency estimation techniques, signal analysis considers the use of eigenvalues and eigenvectors of the correlation matrix for the purposes of defining signal and noise subspaces. In practice, we estimate the signal and noise subspaces by using the eigenvectors and eigenvalues of the sample correlation matrix, as two orthogonal subspaces. The MUSIC algorithm is a method for the estimation of pseudospectrum of signal which is based on covariance attributes and eigenspace. In this method, input signal is modeled as

$$
x(n)=\sum_{p=1}^{P} \alpha_{p} e^{j \omega_{p} n}+w(n)
$$

where $\omega_{p}$ is a normalized angular frequency of the components, and $w$ is additive white noise. Since we will make use of matrix methods based on a certain time window of length $M$, characterizationofthe signal model in the form of a vector over this time window would be useful. The time window includes the sample delays of the signal. It can be written as

$$
x(n)=[x(n) x(n+1) \cdots x(n+m-1)]^{T} .
$$

We can then write the signal model consisting of complex exponentials in noise from (2) for a length- $M$ time-window vector as

$$
x(n)=\sum_{p=1}^{P} \alpha_{p} v\left(\omega_{p}\right) e^{j n \omega_{p}}+w(n)=s(n)+w(n),
$$

where $w(n)=[w(n) w(n+1) \cdots w(n+m-1)]^{T}$ is the time-window vector of white noise and

$$
v(\omega)=\left[\begin{array}{ll}
1 & e^{j \omega} \cdots e^{j \omega(M-1)}
\end{array}\right]^{T}
$$

is the time-window frequency vector. Consider the timewindow vector model consisting of a sum of complex exponentials in noise from (4). The autocorrelation matrix of this model can be written as the sum of signal and noise autocorrelation matrices:

$$
\begin{aligned}
R_{x} & =E\left\{x(n) x(n)^{H}\right\} \\
& =R_{s}+R_{w} \\
& =\sum_{p=1}^{P}\left|\alpha_{p}\right|^{2} v\left(\omega_{p}\right) v^{H}\left(\omega_{p}\right)+\sigma_{w}^{2} I \\
& =V A V^{H}+\sigma_{w}^{2} I,
\end{aligned}
$$

where

$$
V=\left[\begin{array}{ll}
v\left(\omega_{1}\right) & v\left(\omega_{2}\right) \cdots v\left(\omega_{p}\right)
\end{array}\right]
$$

is an $M \times P$ matrix and

$$
A=\left[\begin{array}{cccc}
\left|\alpha_{1}\right|^{2} & 0 & \cdots & 0 \\
0 & \left|\alpha_{2}\right|^{2} & \cdots & 0 \\
0 & \cdots & \cdots & 0 \\
0 & \cdots & 0 & \left|\alpha_{p}\right|^{2}
\end{array}\right]
$$

is a diagonal matrix of the powers of each of the respective complex exponentials. The autocorrelation matrix can also be written in terms of its eigen-decomposition:

$$
R_{x}=\sum_{m=1}^{M} \lambda_{m} q_{m} q_{m}^{H}=Q \Lambda Q^{H}
$$

where $\lambda_{m}$ are the eigenvalues in descending order, that is, $\lambda 1 \geq \lambda 2 \geq \cdots \geq \lambda M$ and $\mathbf{q} m$ are their corresponding eigenvectors. Here, $\Lambda$ is a diagonal matrix made up of the eigenvalues found in descending order on the diagonal, while the columns of $\mathbf{Q}$ are the corresponding eigenvectors. The 


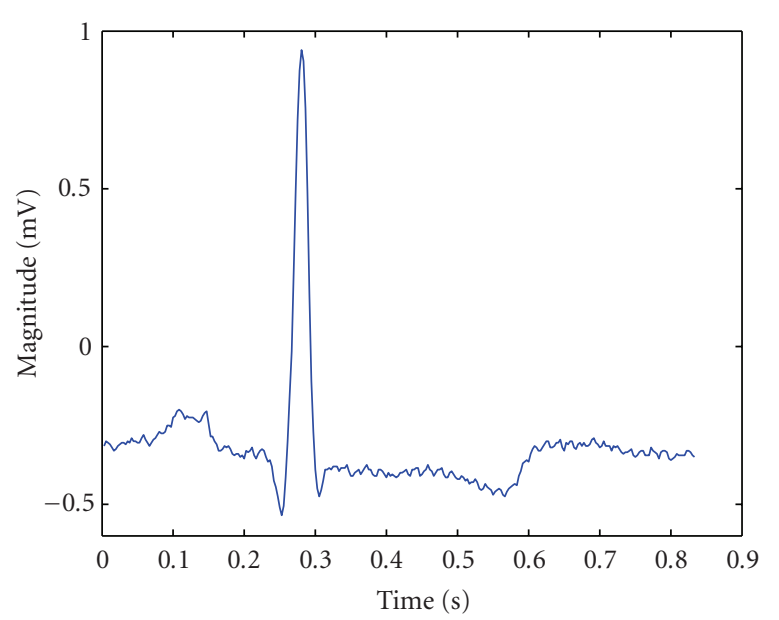

(a)

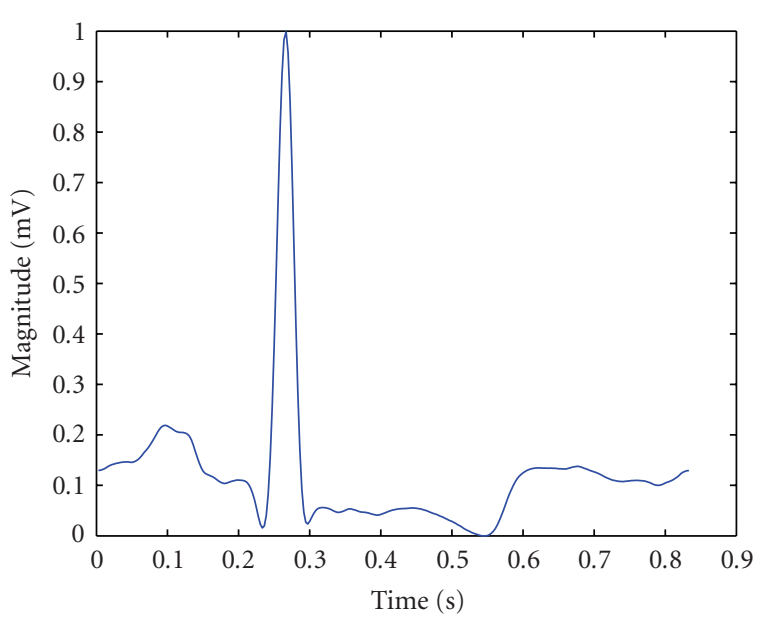

(b)

Figure 2: (a) An ECG pattern and (b) its processed form.

eigenvalues due to the signals can be written as the sum of the signal power in the time window and the noise:

$$
\lambda_{m}=M\left|\alpha_{m}\right|^{2}+\sigma_{m}^{2} \text { for } m \leq P .
$$

The remaining eigenvalues are due to the noise only, that is,

$$
\lambda_{m}=\sigma_{m}^{2} \text { for } m \leq P .
$$

Therefore, the $P$ largest eigenvalues correspond to the signal made up of complex exponentials and the remaining eigenvalues have equal value and correspond to the noise. Thus, we can partition the correlation matrix into portions; due to the signal and noise eigenvectors,

$$
\begin{gathered}
R_{x}=Q_{S} \Lambda Q_{s}^{H}+\sigma_{w}^{2} Q_{w} Q_{w}^{H}, \\
Q_{s}=\left[\begin{array}{ll}
q_{1} & q_{2} \cdots q_{3}
\end{array}\right] \quad Q_{w}=\left[q_{P+1} \cdots q_{M}\right]
\end{gathered}
$$

are matrices whose columns consist of the signal and noise eigenvectors, respectively. From (4), $x(n)$ can be split into two subspaces spanned by the signal and noise eigenvectors, respectively. Since, the eigenvectors of the Hermitian symmetric matrix are orthogonal and the correlation matrix is the Hermitian symmetric, then these two subspaces, known as the signal subspace and the noise subspace, are orthogonal to each other. Therefore, with the projection matrix from an $M$-dimensional space onto an $L$-dimensional subspace $(L<M)$ spanned, we can write the matrices that project an arbitrary vector onto the signal and noise subspaces as

$$
P_{s}=Q_{s} Q_{s}^{H} \quad P_{w}=Q_{w} Q_{w}^{H},
$$

since the eigenvectors of the correlation matrix are orthonormal. Since the two subspaces are orthogonal

$$
P_{w} Q_{s}=0 \quad P_{s} Q_{w}=0,
$$

then all the time-window frequency vectors from (4) must lie completely in the signal subspace, that is,

$$
P_{s} v\left(\omega_{p}\right)=v\left(\omega_{p}\right) \quad P_{w} v\left(\omega_{p}\right)=0 .
$$

Because of the orthogonality between the noise and signal subspaces, all the time-window frequency vectors of the complex exponentials are orthogonal to the noise subspace from (15). Thus, for each eigenvector $(P<m \leq M)$,

$$
v^{H}\left(\omega_{p}\right) q_{m}=\sum_{k=1}^{M} q_{m}(k) e^{-j \omega_{p}(k-1)}=0
$$

for all the $P$ frequencies $\omega_{p}$ of the complex exponentials. Therefore, if we compute a pseudospectrum for each noise eigenvector as

$$
\hat{R}_{m}(\omega)=\frac{1}{\left|v^{H}(\omega) q_{m}\right|^{2}}=\frac{1}{|Q m(\omega)|^{2}},
$$

where the polynomial $Q m(\omega)$ has $M-1$ roots, $P$ of which correspond to the frequencies of the complex exponentials. These roots produce $P$ peaks in the pseudospectrum from (17). Note that the pseudospectra of all $M$-P noise eigenvectors share these roots that are due to the signal subspace. The remaining roots of the noise eigenvectors, however, occur at different frequencies. There are no constraints on the location of these roots, so that some may be close to the unit circle and produce extra peaks in the pseudospectrum. A means of reducing the levels of these spurious peaks in the pseudospectrum is to average the $M-P$ pseudospectra of the individual noise eigenvectors:

$$
\widehat{R}_{\text {MUSIC }}(\omega)=\frac{1}{\sum_{m=P+1}^{M}\left|v^{H}(\omega) q_{m}\right|^{2}}=\frac{1}{\sum_{m=P+1}^{M}|Q m(\omega)|^{2}},
$$

which is known as the MUSIC pseudospectrum. The frequency estimates of the $P$ complex exponentials are then taken as the $P$ peaks in this pseudospectrum. The term pseudospectrum is used because the quantity in (18) does not contain information about the powers of the complex exponentials or the background noise level [13]:

$$
\widehat{R}_{\mathrm{MUSIC}}(\omega)=\frac{1}{V^{H}(\omega) Q_{w} Q_{w}^{H} V(\omega)},
$$


where the variable frequency vector or frequency scanning vector is $V(\omega)$ defined in (5). The varied range for angular frequency $\omega$ is $[-\pi, \pi]$. The product $Q_{w} Q_{w}^{H}$ represents a projection matrix on the noise subspace [12]. Because the signal is real, the pseudospectrum is just calculated for $0-\pi$ frequencies, and then, a 129 frequency samples pseudospectrum is obtained by taking the sample space of $\pi / 128$ including zero.

\subsubsection{Feature Vector Computation}

In the presence of two or more classes, feature extraction will be a selection of the most effective features for preserving class separability. Class separability criteria are essentially independent of coordinate systems, and are completely different from the criteria for signal representation [19]. In this study, the filtering and scaling described in Sections 2.1.1 and 2.1.2 are applied to enhance signal quality, and to unify patterns. Two sets of features are extracted from the patterns. One set is the spectral features and the other is the morphological features. To estimate spectral features, the MUSIC algorithm is used to compute pseudospectrum of patterns. Because the signal is real, the pseudospectrum is just calculated for $0-\pi$ frequencies, and then a 129 frequency samples pseudospectrum is obtained.

Since the high-frequency signals were filtered in the preprocessing module, they are of no value in extraction of features. Empirically, we come to the conclusion that using the first 28 frequency samples of the pseudospectrum produces the best results. Examples of pseudospectra of heartbeats in the nine classes are shown in Figure 3. It is obvious that the last frequency samples of these pseudospectra are similar and cannot be efficient features.

The ECG signal reflects the electrical activity of the heart and the P-QRS-T segment represents one full cardiac cycle in the time-domain. The magnitude and location of the $P$, QRS, and $T$ waves are indicative of the heart functioning. The arrhythmias are pathological changes in the P-QRS-T segment, as well as in the heart rate. Therefore, the variances of the signal peaks values and times are also important feature for characterizing arrhythmias. The location and magnitude of the peaks in each heart cycle characterize the function of the heart.

In order to find the peaks, first, the gradient of a scaled heart cycle is calculated. The gradient of an ECG signal in the plane containing the $t$ (time) and $m$ (magnitude) axes is generally represented by the symbol $\nabla$, and is defined as the change in the $m$ coordinate divided by the corresponding change in time. This is shown as follows:

$$
\nabla x(t)=\frac{x(t+\Delta t)-x(t)}{\Delta t},
$$

where the value of $t$ is started from 0 until the end of the given samples, and $\Delta t$ is set to be 1 .

Second, all the peaks in any single heart cycle which are related to the P-QRS-T segments are determined by locating the zeros of the gradient. It is obvious that a heart cycle may not produce all segment peaks depending on the patient condition. Now, the variances of the magnitude and time of those peaks obtained in the given heart cycle are computed. These features as well as spectral features are then built into a feature vector used as the input of the neural networks used later.

It is essential to perform a normalization process in order to standardize all the features to the same leve because the quantity of the features may be quite different. The formula of the normalization is defined as follows:

$$
x_{i j}^{\prime}=\frac{x_{i j}-\min }{\max -\min },
$$

where $x_{i j}$ is the $j$ th component of the $i$ th feature vector, min and max are the minimum and maximum, respectively, of the $j$ th component of the feature vectors. It maps a wideranged signal to a limited range of $[0,1]$. In our experiment, the minimum and the maximum of each component in the feature vectors are calculated from the training dataset and are used throughout the experiments. The normalized feature vectors serve as inputs to the following neural network classifiers.

\subsection{Classification by neural networks}

To classify nine different types of arrhythmias, two neural networks are used. Following, these two are discussed in detail.

\subsubsection{Multilayer perceptrons}

An important class of neural networks is multilayer feedforward networks. Usually, the network is comprised of a set of sensory units (source nodes) that form the input layer. It also includes one or more hidden layers of computation nodes and an output layer of computation nodes. Throughout the neural network, the input signal propagates in a forward direction and on a layer-by-layer basis. Since they are meant to be a generalization of the single-layer perceptron, these neural networks are referred to as multilayer perceptrons (MLPs).

Multilayer perceptrons are trained in a supervised manner algorithm with highly popular algorithm known as error backpropagation algorithm whose basis is on the errorcorrection learning rule. As such, it may be conceived of a generalization of an equally popular adaptive filtering algorithm.

Error backpropagation learning is a two-pass transmission through the layers of the networks. The passes are forward and backward. In the forward pass, following the application of an activity pattern (input vector) to the sensory nodes, its effect propagates through the network layer by layer with the result of production of a set of outputs as the actual response of the network. During the forward pass, the synaptic weights of the networks are fixed. However, during the backward pass, an error-correction rule is used to adjust the synaptic weights. A major part is the subtraction of the actual response from a desired (target) response to make an error signal. By the backward propagation of this error signal against the direction of synaptic connection, "errorbackpropagation" is done. An Adjustment of the synaptic 


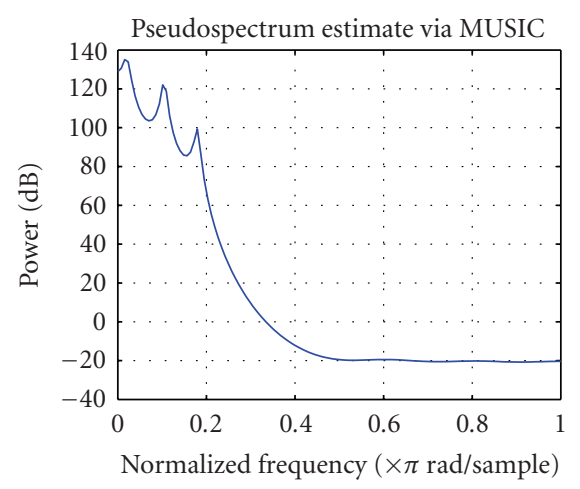

(a) NORMAL

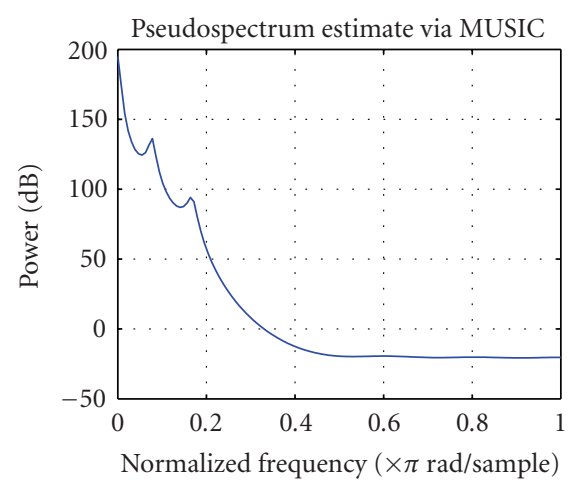

(d) PVC

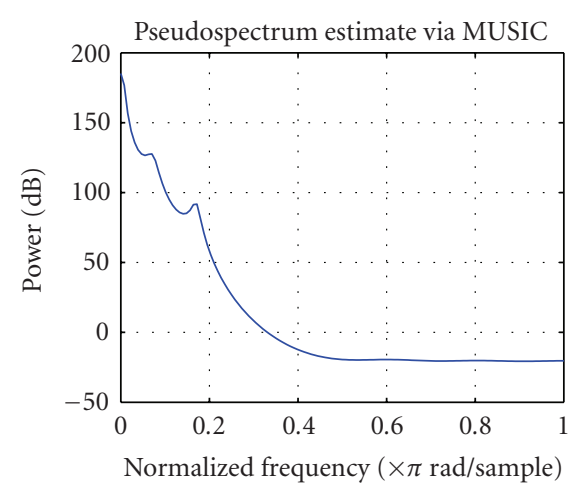

(g) PACE

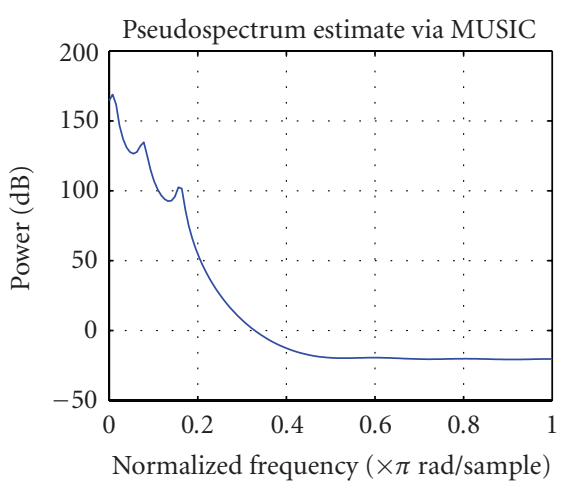

(b) LBBB

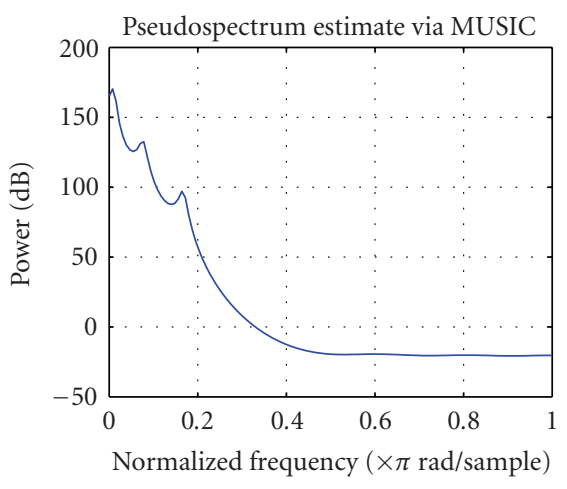

(e) FUSION

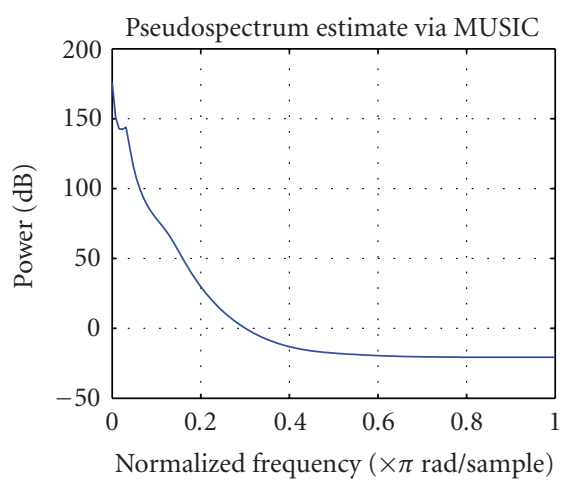

(h) FLAW

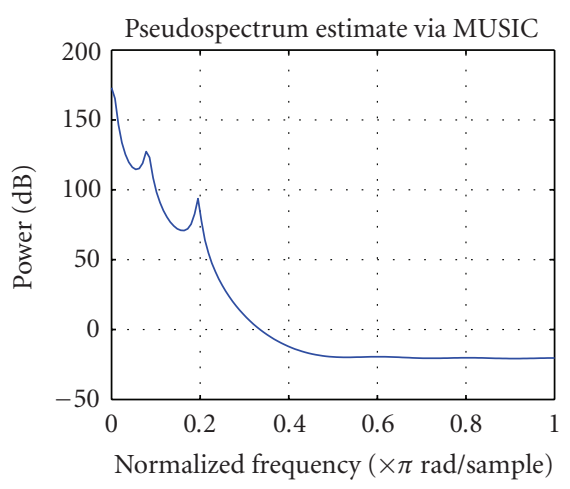

(c) RBBB

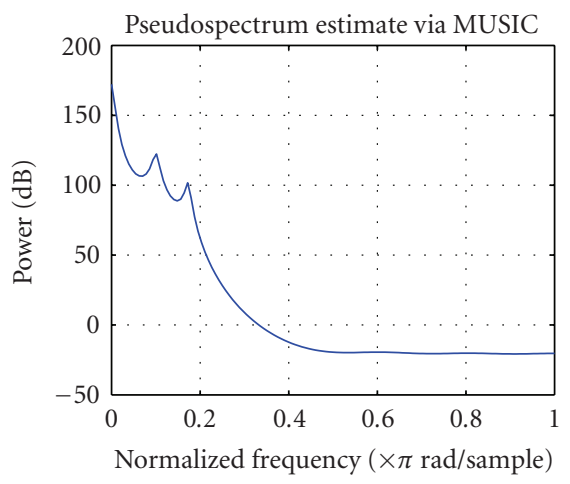

(f) APC

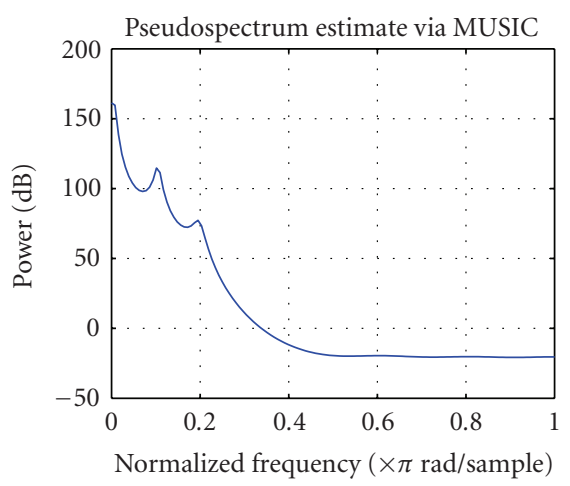

(i) PFUS

FIgURE 3: Pseudospectrum of each class which was estimated by MUSIC algorithm.

weights is done to move the actual response of the network closer to the desired response in a statistical way. The error backpropagation algorithm is also referred to in the literature as the backpropagation algorithm, or simply backprop. The learning process performed with the algorithm is called backpropagation learning. Figure 4 depicts a portion of the multilayer perceptron. Two kinds of signals are identified in this network [20].

\subsubsection{Probabilistic neural network}

For classification problems, we use probabilistic neural networks (PNNs) with straightforward and trainingindependent designs. If given enough data, these networks guarantee coverage to the Bayesian classifier. These networks have a two-layer structure and generalize well.

The first layer function is to produce a vector possessing elements indicative of the degree of closeness of a presented input to a training input. It performs this by computing the distances from the input vector to the training input vectors. Then the second layer produces its own net output of a network of probabilities by summing these contributions for each class of input. The sensitivity of the radial basis neurons can be adjusted by varying the value of smoothing factor. In the study, we empirically determine the value of smoothing factor as 0.08 that produces satisfactory results. Finally, a complete transfer function on the output of the second layer picks the maximum of these probabilities, and produces a 1 


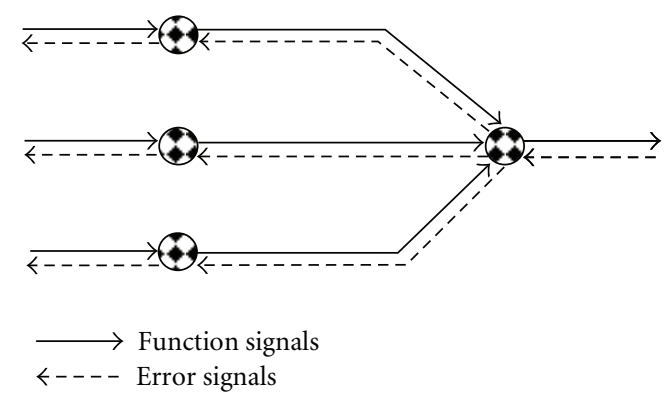

FIGURE 4: Illustration of the directions of two basic signal flows in a multilayer perceptron: forward propagation of function signals and backpropagation of error signals [20].

for that class and a 0 for the other classes [21]. The number of neurons in the second layer is the same as that of the desired classes, that is, nine neurons in the competition layer in our experiments.

\section{EXPERIMENT DESIGN}

This study involved 22 ECG records from the MIT-BIH arrhythmia database. Each MIT/BIH record is 30 minutes in duration, includes two leads, and is annotated throughout; by this we mean that each beat (QRS complex) is described by a label called an annotation [2]. The sampling frequency is $360 \mathrm{~Hz}$.

The nine beat types employed in the study were normal beat (NORMAL), left bundle branch block beat (LBBB), right bundle branch block beat (RBBB), premature ventricular contraction (PVC), fusion of ventricular and normal beat (FUSION), atrial premature contraction (APC), paced beat (PACE), ventricular flutter wave (FLWAV), and fusion of paced and normal beat (PFUS). Testing and training sets are separately formed by choosing 13950 vectors. For each class, the numbers of training and testing sets are equal. Testing and training sets are formed by data obtained from 17 records of MIT-BIH arrhythmia database. The sources of the ECG beats are shown in Table 1. We randomly select the given number of training and testing heartbeat patterns from the selected ECG recordings.

In our experiments, we used the multilayer perceptron (MLP) and the probabilistic neural network (PNN) as the pattern classifiers. The original dataset was separately divided into training and testing groups. Two factors that might affect the efficiency of MLP are the number of hidden layer neurons and synapses initial weight. Experiments were performed to test the effects of the initial weight and the number of hidden layer neurons parameter. One factor that might affect the efficiency of the PNN is the smoothing factor, which is the standard deviation of the Gaussian kernel. Experiments were also performed to test the effect of the smoothing parameter.

The performances of the classification are evaluated in terms of sensitivity, specificity, and overall accuracy. Sensitivity and specificity are used to evaluate the ability of the classification system to discriminate one class against the other. The sensitivity is calculated as the proportion of
TABLE 1: The sources and the number of ECG samples used in this study.

\begin{tabular}{lccc}
\hline Type & $\begin{array}{c}\text { MIT/BIH } \\
\text { file number }\end{array}$ & $\begin{array}{c}\text { Training } \\
\text { (no./file) }\end{array}$ & $\begin{array}{c}\text { Testing } \\
\text { (no./file) }\end{array}$ \\
\hline NORMAL & $100,103,106,202,209$ & 300 & 300 \\
LBBB & $109,111,207$ & 300 & 300 \\
RBBB & $118,124,212,231$ & 300 & 300 \\
PVC & 106,119 & 200 & 200 \\
FUSION & 200,208 & 300 & 300 \\
& 208,213 & 150 & 150 \\
APC & 118,207 & 40 & 40 \\
& 209,222 & 100 & 100 \\
PACE & 232 & 300 & 300 \\
FLWAV & $102,104,107,217$ & 200 & 200 \\
PFUS & 207 & 235 & 235 \\
& 104 & 330 & 330 \\
Total & 217 & 130 & 130 \\
\hline
\end{tabular}

positive samples correctly assigned to the positive class. The specificity is the proportion of negative samples correctly assigned to the negative class. The overall accuracy is the fraction of the total number of beats correctly classified.

\section{EXPERIMENTAL RESULTS}

The classification results using MLP and PNN are summarized in Table 2. The diagonal elements in the table are the number of correctly classified beats of specific ECG types using the proposed method. In Table 2, MLP neural network generally provides adequate recognition throughout all categories. In each classifier, the lower recognition was resulted for PFUS arrhythmia type, which means that the proposed feature vector has a lower discrimination for them. The system always recognizes NORMAL class correctly and never misclassifies any other classes as NORMAL. In other words, the specificity and sensitivity are 100\% for NORMAL class. The results of MLP classifier are more uniform than PNN classifier. The varied range for MLP is [90.94-100] and for PNN is [79.02-100] . It is obvious that MLP classifier has a higher recognition than PNN classifier for the proposed feature vectors.

Table 3 shows the average and standard deviations of sensitivity, specificity, and overall accuracy of the extracted feature set with MLP and PNN classifiers. For the values in Table 3, we choose the average of the results from 20 trials. The results are computed when the number of MLP hidden layer neurons is 90 and the smoothing factor for PNN is 0.11 . Average of specificity is above $99.57 \%$ for MLP classifier and above $99.11 \%$ for PNN classifier. The average of overall accuracy is $99.03 \%$ and $97.75 \%$ for MLP and PNN classifiers, respectively.

To study the effect of the number of MLP hidden layer neurons in differentiation of the nine ECG beat types, we varied the numbers of hidden layer neurons from 5 to 100 and their effects were considered. Since weight initialization 
TABLE 2: Classification results of the proposed scheme with MLP and PNN.

\begin{tabular}{|c|c|c|c|c|c|c|c|c|c|c|}
\hline & $\begin{array}{l}\text { Output } \\
\text { desired }\end{array}$ & NORMAL & LBBB & RBBB & PVC & APC & PACE & FUSION & FLWAV & PFUS \\
\hline \multirow{9}{*}{$\begin{array}{l}\text { MLP with } 90 \\
\text { neuron in } \\
\text { hidden layer }\end{array}$} & NORMAL & 1500 & 0 & 0 & 0 & 0 & 0 & 0 & 0 & 0 \\
\hline & LBBB & 1 & 897 & 0 & 0 & 0 & 0 & 0 & 0 & 2 \\
\hline & RBBB & 0 & 2 & 1194 & 0 & 1 & 0 & 0 & 0 & 3 \\
\hline & PVC & 0 & 1 & 1 & 990 & 0 & 0 & 0 & 2 & 6 \\
\hline & APC & 0 & 0 & 0 & 0 & 580 & 0 & 0 & 0 & 0 \\
\hline & PACE & 0 & 3 & 0 & 1 & 0 & 793 & 0 & 1 & 2 \\
\hline & FUSION & 0 & 0 & 0 & 0 & 0 & 0 & 300 & 0 & 0 \\
\hline & FLWAV & 0 & 2 & 0 & 2 & 0 & 1 & 0 & 222 & 8 \\
\hline & PFUS & 0 & 2 & 0 & 0 & 0 & 0 & 0 & 0 & 458 \\
\hline \multirow{9}{*}{$\begin{array}{l}\text { PNN with } \\
\text { smoothing } \\
\text { factor } 0.11\end{array}$} & NORMAL & 1500 & 0 & 0 & 0 & 0 & 0 & 0 & 0 & 0 \\
\hline & LBBB & 0 & 894 & 2 & 0 & 0 & 2 & 0 & 0 & 2 \\
\hline & $\mathrm{RBBB}$ & 0 & 0 & 1185 & 3 & 1 & 5 & 0 & 0 & 6 \\
\hline & PVC & 0 & 4 & 1 & 970 & 3 & 12 & 0 & 6 & 4 \\
\hline & APC & 0 & 0 & 0 & 0 & 580 & 0 & 0 & 0 & 0 \\
\hline & PACE & 0 & 1 & 3 & 3 & 0 & 793 & 0 & 0 & 0 \\
\hline & FUSION & 0 & 1 & 0 & 0 & 0 & 1 & 296 & 2 & 0 \\
\hline & FLWAV & 0 & 2 & 0 & 7 & 0 & 3 & 7 & 183 & 33 \\
\hline & PFUS & 0 & 1 & 0 & 0 & 0 & 0 & 0 & 0 & 459 \\
\hline
\end{tabular}

TABLE 3: Classification results of the proposed scheme with MLP and PNN.

\begin{tabular}{lccccc}
\hline & & \multicolumn{2}{c}{ MLP classifier } & \multicolumn{2}{c}{ PNN classifier } \\
& & Average & STD & Average & STD \\
\hline \multirow{5}{*}{ SORMAL } & 100.00 & 0.00 & 100.00 & 0.00 \\
& LBBB & 99.46 & 0.11 & 99.15 & 0.04 \\
& RBBB & 99.19 & 0.21 & 97.90 & 0.20 \\
& PVC & 97.57 & 0.64 & 90.89 & 0.39 \\
& APC & 99.88 & 0.10 & 100.00 & 0.00 \\
& PACE & 99.17 & 0.20 & 99.11 & 0.06 \\
& FUSION & 99.65 & 0.40 & 98.11 & 0.00 \\
& FLWAV & 90.94 & 1.67 & 79.02 & 0.52 \\
Specificity (\%) & PFUS & 99.66 & 0.35 & 99.87 & 0.11 \\
\hline & NORMAL & 100.00 & 0.00 & 100.00 & 0.00 \\
& LBBB & 99.81 & 0.05 & 99.84 & 0.00 \\
& RBBB & 99.97 & 0.02 & 99.86 & 0.02 \\
& PVC & 99.95 & 0.03 & 99.75 & 0.03 \\
& APC & 99.96 & 0.02 & 99.92 & 0.00 \\
& PACE & 99.97 & 0.02 & 99.52 & 0.02 \\
& FUSION & 99.95 & 0.04 & 99.90 & 0.01 \\
\hline Overall accuracy (\%) & FLWAV & 99.87 & 0.03 & 99.83 & 0.02 \\
\hline & PFUS & 99.57 & 0.07 & 99.11 & 0.00 \\
\hline & 99.03 & 0.07 & 97.75 & 0.02 \\
\hline
\end{tabular}

is random, we repeated each of the experiment setups 20 times, and the results were averaged and showed in Figure 5. The standard deviations of overall accuracies for different hidden layer neurons are depicted in Figure 6.

In Figure 5, the discrimination power of MLP, revealed as the average overall accuracy, increases rapidly at small

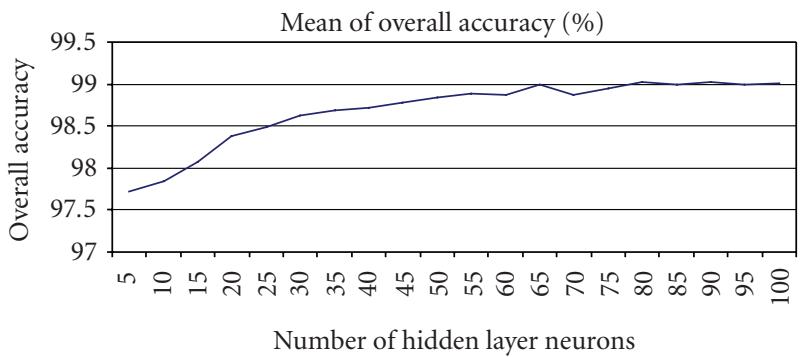

FIGURE 5: Effect of hidden layer neuron number on average overall accuracy (\%) with multilayer perceptron.

numbers of hidden layer neurons and then reaches a plateau at around 90 hidden layer neurons. At even higher hidden layer neuron numbers, the average overall accuracies stay at around 99\%. Further increase in hidden layer neuron number does not significantly increase the accuracy of the classifier. On the other hand, in Figure 6, the standard deviations of averaged overall accuracies decrease until 75 hidden layer neurons and reach a plateau at range [75-90] and then have an ascending approach. Figures 5 and 6 show that the best number of hidden layer neurons is 90 .

The smoothing factor of the Gaussian kernel function is a control parameter of the probabilistic neural network. To study the effect of the smoothing factor to the performance of the PNN classifier, a series of experiments was conducted and the results were shown in Figure 7. The results show that when the smoothing factor increases, the overall accuracy decreases. When the smoothing factor is chosen in the range from 0.04 to 0.11 , the overall accuracies are the highest. 


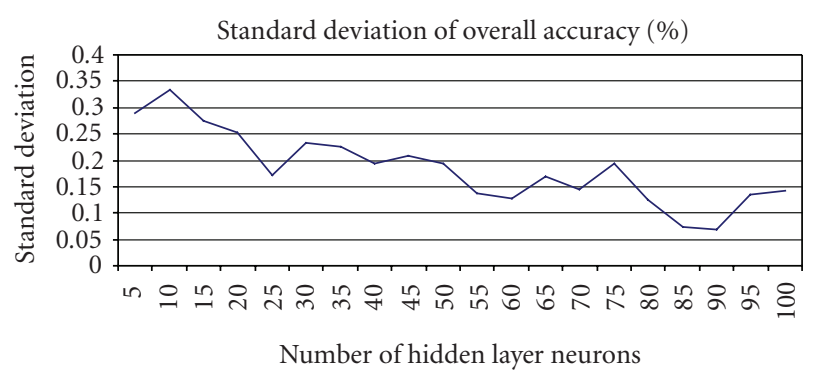

FIGURE 6: Standard deviation of overall accuracy versus number of hidden layer neurons of multilayer perceptron.

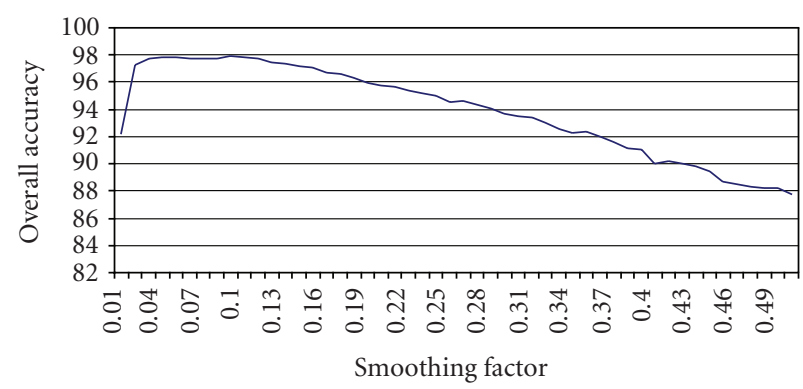

FIGURE 7: Effects of different smoothing factors in the probabilistic neural network.

Where the smoothing factor value is not mentioned, it is 0.11 .

\section{DISCUSSIONS}

Comparing the average accuracy achieved with the two neural network classifiers depicted in Table 3, the result reveals that the MLP neural network has better performance than PNN neural network to integrate with extracted features. It is also interesting to compare the result of proposed method with other heartbeat recognition systems presented in the literature. Although a lot of studies dealing with heartbeat classification are present in the literature, a strict comparison with the results of the present work is difficult to perform, since different heartbeat categories were used and different ECG datasets were considered. The following methods which form representative heartbeat classifications are chosen for this comparison: a modified mixture of experts network structure for ECG beats classification with diverse features (MME) [22]; comparing binary and realvalued coding in hybrid immune algorithm for feature selection and classification of ECG signals (HIA) [23]; ECG beat classification by a novel hybrid neural network (NHNN) [9]; integration of independent component analysis and neural networks for ECG beat classification (ICANN) [11]; ECG beat classification using mirrored Gauss model (MGM) [24]; the comparison of different feedforward neural network architectures for ECG signal diagnosis (DFFNN) [25]. Table 4 compares the accuracy of these systems, in which the first row of the table is the result of the method, combining the extracted features and MLP classifier proposed in this
TABLE 4: Comparative results of different ECG beat classification methods.

\begin{tabular}{lcc}
\hline Methods & Number of beat types & Accuracy $(\%)$ \\
\hline Proposed & $\mathbf{9}$ & $\mathbf{9 9 . 0 3}$ \\
MME & 5 & 97.78 \\
HIA & 6 & 97.9 \\
NHNN & 10 & 96 \\
ICANN & 8 & 98 \\
MGM & 2 & 93.94 \\
DFFNN & 6 & 93 \\
\hline
\end{tabular}

paper. All methods that were mentioned in the table use local learning sets taken from the same sources as the selected testing sets. Since different numbers of beat types were exploited in different systems, the averaged classification accuracy was calculated for comparison. Although this comparison may not be completely fair, the proposed system seems to be a powerful tool to use as ECG beat classification system.

The result shows that our proposed method provides relatively higher classification accuracy than the other systems. The main advantage of our proposed method is that it always classifies NORMAL classes correctly and never misclassifies any as NORMAL, the feature other methods lack. This implies that in clinical examinations, the system always alerts of existing arrhythmic problems correctly.

\section{CONCLUSION}

In this paper, we proposed a scheme based on multiple signal classification algorithm, two morphological descriptors, and neural networks to classify nine ECG arrhythmia signals. MUSIC algorithm is used to compute pseudospectrum of ECG signals. The first 28 frequency samples of the pseudospectrum with variance of peak values and times are combined as feature vector and then served as input for the following neural network classifiers. Two neural networks, including multilayered perceptron (MLP) and probabilistic neural network (PNN), were employed in the study and their effects were compared. Of the two neural network classifiers, MLP shows a slightly better performance than PNN in terms of overall accuracy. The mean of overall accuracy with 90 neurons in hidden layer was $99.03 \%$. Because the system can recognize NORMAL class with $100 \%$ of specificity and sensitivity, we can use it with high confidence. The result shows that the proposed feature vector can well show the ECG signals nature and function. This study proves that the proposed method is an excellent model for the computeraided diagnosis of heart diseases based on ECG signals.

\section{REFERENCES}

[1] M. Wiggins, A. Saad, B. Litt, and G. Vachtsevanos, "Evolving a Bayesian classifier for ECG-based age classification in medical applications," Applied Soft Computing Journal, vol. 8, no. 1, pp. 599-608, 2008. 
[2] G. B. Moody, WFDB Programmer's Guide, PhysioNet, Cambridge, Mass, USA, 2006.

[3] H. D. Kim, C. H. Min, and T. S. Kim, "Adaptable noise reduction of ECG signals for feature extraction," in Proceedings of the 3rd International Symposium on Neural Networks (ISNN '06), vol. 3973 of Lecture Notes in Computer Science, pp. 586-591, Chengdu, China, May 2006.

[4] T. Y. Lo and P. C. Tang, "A fast bandpass filter for ECG processing," in Proceedings of the 4th Annual International Conference of the IEEE Engineering in Medicine and Biology Society (EMBS '82), pp. 20-21, Philadelphia, Pa, USA, September 1982.

[5] S.-N. Yu and K.-T. Chou, "A switchable scheme for ECG beat classification based on independent component analysis," Expert Systems with Applications, vol. 33, no. 4, pp. 824-829, 2007.

[6] P. Tadejko and W. Rakowski, "Mathematical morphology based ECG feature extraction for the purpose of heartbeat classification," in Proceedings of the 6th International Conference on Computer Information Systems and Industrial Management Applications (CISIM '07), pp. 322-327, Elk, Poland, June 2007.

[7] I. Jekova, G. Bortolan, and I. Christov, "Assessment and comparison of different methods for heartbeat classification," Medical Engineering \& Physics, vol. 30, no. 2, pp. 248-257, 2008.

[8] R. Ceylan and Y. Özbay, "Comparison of FCM, PCA and WT techniques for classification ECG arrhythmias using artificial neural network," Expert Systems with Applications, vol. 33, no. 2, pp. 286-295, 2007.

[9] Z. Dokur and T. Ölmez, "ECG beat classification by a novel hybrid neural network," Computer Methods and Programs in Biomedicine, vol. 66, no. 2-3, pp. 167-181, 2001.

[10] P. de Chazal and R. B. Reilly, "A comparison of the ECG classification performance of different feature sets," in Proceedings of the 27th Annual Conference on Computers in Cardiology, pp. 327-330, Cambridge, Mass, USA, September 2000.

[11] S.-N. Yu and K.-T. Chou, "Integration of independent component analysis and neural networks for ECG beat classification," Expert Systems with Applications, vol. 34, no. 4, pp. 2841-2846, 2008.

[12] S. Haykin, Adaptive Filter Theory, Prentice-Hall, Englewood Cliffs, NJ, USA, 2nd edition, 1991.

[13] D. G. Manolakis, V. K. Ingle, and S. M. Kogon, Statistical and Adaptive Signal Processing, Artech House, Boston, UK, 2005.

[14] T. Iwata, Y. Goto, and H. Susaki, "Application of the multiple signal classification (MUSIC) method for one-pulse burstecho Doppler sonar data," Measurement Science and Technology, vol. 12, no. 12, pp. 2178-2184, 2001.

[15] T. K. Moon and W. C. Stirling, Mathematical Methods and Algorithms for Signal Processing, Prentice-Hall, Englewood Cliffs, NJ, USA, 2000.

[16] A. Hirata, E. Taillefer, H. Yamada, and T. Ohira, "Handheld direction of arrival finder with electronically steerable parasitic array radiator using the reactance-domain MUltiple SIgnal Classification algorithm," IET Microwaves, Antennas \& Propagation, vol. 1, no. 4, pp. 815-821, 2007.

[17] "MIT/BIH arrhythmia database directory," Tech. Rep. BMEC TR010, Division of Health Sciences and Technology, Harvard University and Massachusetts Institute of Technology, Cambridge, Mass, USA, July 1992, CD-ROM.

[18] F. M. Ham and S. Han, "Classification of cardiac arrhythmias using fuzzy ARTMAP," IEEE Transactions on Biomedical Engineering, vol. 43, no. 4, pp. 425-430, 1996.
[19] K. Fukunaga, Introduction to Statistical Pattern Recognition, Academic Press, San Diego, Calif, USA, 2nd edition, 1990.

[20] S. Haykin, Neural Networks a Comprehensive Foundation, Prentice-Hall, Englewood Cliffs, NJ, USA, 1999.

[21] H. Demuth and M. Beale, Neural Network Toolbox, MathWorks, Natick, Mass, USA, 2004.

[22] I. Güler and E. D. Übeyli, "A modified mixture of experts network structure for ECG beats classification with diverse features," Engineering Applications of Artificial Intelligence, vol. 18, no. 7, pp. 845-856, 2005.

[23] M. Bereta and T. Burczyński, "Comparing binary and realvalued coding in hybrid immune algorithm for feature selection and classification of ECG signals," Engineering Applications of Artificial Intelligence, vol. 20, no. 5, pp. 571585, 2007.

[24] Q. Zhou, X. Liu, and H. Duan, "ECG beat classification using mirrored gauss model," in Proceedings of the 27th Annual International Conference of the IEEE Engineering in Medicine and Biology Society (EMBS '05), pp. 5587-5590, Shanghai, China, September 2005.

[25] H. G. Hosseini, D. Luo, and K. J. Reynolds, "The comparison of different feed forward neural network architectures for ECG signal diagnosis," Medical Engineering and Physics, vol. 28, no. 4, pp. 372-378, 2006. 